\title{
Comparison of an enzyme immunoassay for the detection of Helicobacter pylori antigens in the faeces with the urea breath test
}

Ashley J Shepherd, Craig L Williams, Conor P Doherty, Margaret Hossack, Tom Preston, Kenneth E L McColl, Lawrence T Weaver

\begin{abstract}
Background-Current diagnostic tests for Helicobacter pylori are invasive (endoscopy) or indirect (urea breath test, serology).

Aims-To evaluate a new enzyme immunoassay (EIA) which detects $H$ pylori antigens in faeces, by comparing its sensitivity and specificity in children with the ${ }^{13} \mathrm{C}$ urea breath test (UBT).

Methods-A total of 119 children underwent a UBT and provided a faecal sample for antigen testing within seven days. After an overnight fast each child provided a pretest breath sample, and samples at $\mathbf{3 0}$ and $\mathbf{4 0}$ minutes after ingestion of $100 \mathrm{mg}{ }^{13} \mathrm{C}$ labelled urea. ${ }^{13} \mathrm{C}$ enrichment of breath was measured by isotope ratio mass spectrometry. Faeces were stored at $-70^{\circ} \mathrm{C}$ until antigen testing, using the EIA. Samples were read spectrophotometrically at $450 \mathrm{~nm}$ and results were interpreted using recommended cut offs of optical density $<0.14$ as negative, $\geqslant 0.16$ as positive, with $\geqslant 0.14$ and $<0.16$ representing equivocal results. Sensitivity and specificity were calculated using the manufacturer's cut off compared with UBT.

Results-Sensitivity and specificity were $88 \%$ and $82 \%$, respectively. Negative and positive predictive values were $97 \%$ and $58 \%$.

Conclusions-The EIA is an alternative, non-invasive, and easy to use method for the detection of $\boldsymbol{H}$ pylori in children. Its high negative predictive value suggests a role in screening out uninfected children. (Arch Dis Child 2000;83:268-270)
\end{abstract}

Keywords: Helicobacter pylori; faeces; ELISA; urea breath test

Helicobacter pylori is probably the commonest bacterial infection of humans. The infection is likely to be acquired in childhood, ${ }^{1}$ and in the developing world the incidence of infection in infancy may be up to $50 \% .^{2}$ The rate of new infections in adults in developed countries is low, ${ }^{3}$ and therefore an understanding of the epidemiology and transmission of $H$ pylori infection requires studies to be conducted in children.

The methods of detection of $H$ pylori infection in children are the same as in adults and can broadly be divided into invasive and non-invasive. Invasive methods involve endoscopy and gastric mucosal biopsy with detection of the organism by Campylobacter like organism (CLO) testing, microscopic examination of histological sections, or bacterial culture. Invasive methods are not suitable for large scale population studies.

Non-invasive tests rely either on the detection of gastric bacterial urease activity or on a serological response to the bacterium. There have been numerous serological studies performed in children, and the ${ }^{13} \mathrm{C}$ urea breath test (UBT) has recently been shown, in an epidemiological study in children, to be an alternative to serology and mucosal biopsy. ${ }^{2}$ Another non-invasive test has recently been developed, which relies on the detection, by enzyme immunoassay, of $H$ pylori antigens in the faeces. The test has hitherto been tested only in adults, ${ }^{4}$ but owing to its non-invasive nature it may be suitable for both individual and population studies in children. The aim of the present study was firstly to evaluate the sensitivity and specificity of the new enzyme immunoassay (EIA) test compared with ${ }^{13} \mathrm{C}$ UBT, and secondly to determine the bacteriological specificity of the test.

\section{Materials and methods}

SUBJECTS

All children undergoing a UBT at the Royal Hospital for Sick Children, Glasgow as part of their routine investigation were invited to participate in the study. A total of 119 children were recruited ( 72 boys and 47 girls; mean age 10.4 years, range 3.9-18.4). No children who had received antibiotics, $\mathrm{H}_{2}$ receptor antagonists, or proton pump inhibitors during the three weeks prior to testing were enrolled. The children all provided a faecal sample within seven days of the UBT by mailing a small sample in a clean universal container to the principle investigator.

\section{${ }^{13} \mathrm{C}$ UREA BREATH TEST}

After an overnight fast each child ingested an oral dose of $100 \mathrm{mg}{ }^{13} \mathrm{C}$ urea (99 atom \% excess, CK Gas, Berks, UK), administered in $20 \mathrm{ml}$ of $15 \%$ Polycose (Abbot Labs, Dublin, Eire). Baseline breath samples were collected by asking the child to blow with a straw into a vacutainer, before and at 30 and 45 minutes after ingestion of the labelled urea. ${ }^{13} \mathrm{C}$ enrichment of breath was determined by isotope ratio mass spectrometry; a rise in ${ }^{13} \mathrm{C}$ enrichment of more than $66.0 \mathrm{ppm}$ above fasting baseline 


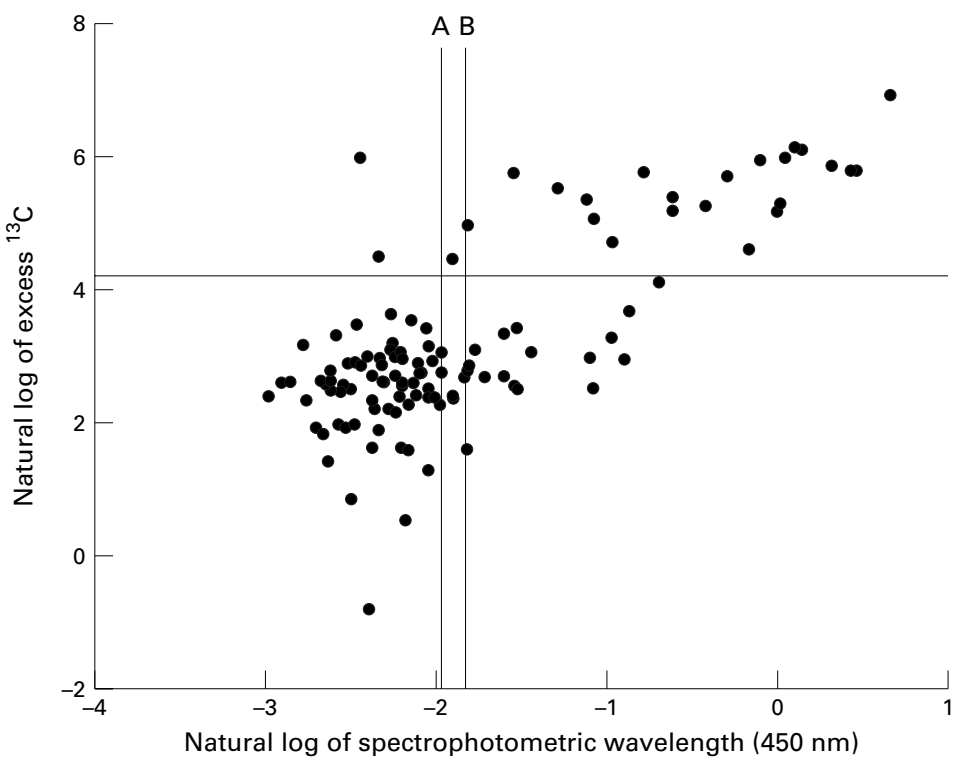

Figure 1 Natural log of EIA result versus natural log of excess ${ }^{13} \mathrm{C}$ UBT result. Cut offs for $\operatorname{EIA}(A=0.14, B=0.16)$ and $U B T(66 \mathrm{ppm})$ are shown.

(relative to a Pee Dee Belemnite (PDB) standard) was regarded as diagnostic of $H$ pylori infection. ${ }^{2}$

\section{ENZYME IMMUNOASSAY}

An enzyme immunoassay (Premier Plantinum HpSA, Meridian Diagnostics Inc., Cincinnati, Ohio, USA) was used to detect $H$ pylori in the stool. It utilises an immunoaffinity purified polyclonal anti- $H$ pylori rabbit antibody absorbed to microwells. Faecal specimens were stored at $-70^{\circ} \mathrm{C}$ until the test was performed. Diluted faecal samples and a peroxidase conjugated polyclonal antibody were added to the wells and incubated for one hour at $24^{\circ} \mathrm{C}$. The wells were washed to remove any unbound material, and a substrate was added before a further 10 minutes of incubation. A stop solution was then added and absorbance was measured spectrophotometrically at $450 \mathrm{~nm}$. The results were assigned to positive, negative, or equivocal groups on the basis of the manufacturer's recommended cut off values.

\section{CROSS REACTIVITY STUDIES}

National collection of type cultures (NCTC) strains of Helicobacter which are closely related to $H$ pylori were used in cross reactivity experiments. They were $H$ mustelae, $H$ canis, $H$ felis, $H$ acinonyx, $H$ pamatensis, $H$ cinaedii, and $H$ fenneliae. All of the organisms were cultured microaerophilically (BBL Campypak) for 48 hours at $37^{\circ} \mathrm{C}$ in brain heart infusion (BHI) broth supplemented with $5 \%$ vol/vol yeast extract and $1 \% \mathrm{vol} / \mathrm{vol}$ horse serum. At 48 hours decimal dilutions of the broths were made in $\mathrm{BHI}$ broth from $10^{-1}$ to $10^{-6}$. The dilutions were mixed with an equal volume of faeces, which had previously been shown to be antigen negative by EIA. Bacterial cultures not mixed with faeces and negative faeces with no added bacteria were tested simultaneously.

The study was approved by the Ethics Committee at the Royal Hospital for Sick Children, Glasgow and performed with informed paren- tal consent. The results were analysed using $\chi^{2}$ analysis using the manufacturers, cut offs for the EIA result. Sensitivity, specificity, positive, and negative predictive values were calculated using standard methods.

\section{Results}

A total of 119 children were tested using the UBT and EIA, and results were available from all (fig 1). Analysis employed a UBT cut off of 66.0 ppm excess ${ }^{13} \mathrm{C}$ and an EIA spectrophotometric absorbance cut off of $<0.14$ negative, $\geqslant 0.16$ positive, and $>0.14$ to $<0.16$ as an equivocal result as recommended by the manufacturers.

Twenty three children were positive and 73 were negative by both UBT and EIA. A further 17 were UBT negative but EIA positive whereas only two were UBT positive and EIA negative. Of the four equivocal EIA results only one was UBT positive. The sensitivity and specificity were calculated as $88 \%$ and $82 \%$ respectively at a cut off of 0.16 , with the positive predictive value of a result $>0.16$ being $58 \%$ and a negative predictive value of a result $<0.14$ being $97 \%$.

\section{BACTERIOLOGICAL STUDIES}

In addition to the bacteriological specificity studies described by the manufacturer, the further cross reactivity of the test was assessed using the range of Helicobacter spp. listed in the methods section. $H$ felis and $H$ acinonyx showed a positive reaction to a dilution of $10^{-4}$ when assayed by the EIA. All others showed no cross reactivity at all dilutions.

\section{Discussion}

The EIA has been shown to be a useful non-invasive diagnostic test for $H$ pylori in children. Using the manufacturer's cut off we calculate the sensitivity and specificity of the test to be $88 \%$ and $82 \%$ respectively compared with ${ }^{13} \mathrm{C}$ UBT and found the test to have positive and negative predictive values of $58 \%$ and $97 \%$. The assay has only recently been made available and this is one of the first published evaluations in children. In previous studies in adults, sensitivity ranged from $89 \%{ }^{5}$ to $96 \%,{ }^{67}$ and specificity from $75 \%{ }^{7}$ to $100 \%{ }^{8}$

To validate the EIA we compared it to the ${ }^{13} \mathrm{C}$ UBT. The diagnosis of $H$ pylori infection can be based on endoscopy with biopsy and culture, but the ${ }^{13} \mathrm{C}$ UBT is also a sensitive indicator of the presence of the bacterium in the stomach and compares well with invasive testing. ${ }^{9}$ We have validated it against mucosal biopsy in a small group of young children, and also developed a statistical method of interpreting the results of the UBT within a large population of infected children. ${ }^{10}$

Most epidemiological studies have been performed using serology, which is less sensitive and specific than the UBT. ${ }^{11}$ When our results from the EIA were compared with commercial serological tests it was clear that while the positive predictive value of the EIA at $58 \%$ was lower than serological tests, which range from 
$87 \%$ to $100 \%,{ }^{12}$ the negative predictive value was equal to the best of the kits (range 80-100\%).

Colonisation with $H$ pylori does not necessarily indicate disease; and tests with a high negative predictive value are useful to exclude infection whereas those with high positive predictive values indicate the need for further investigation. It is important to note that the negative predictive value depends on the cut off value chosen. We used the manufacturer's cut off which is based on adult studies. If the test is used to screen patients, then choosing a cut off to maximise the negative predictive value may be appropriate.

A test which detects $H$ pylori antigen in faeces may be of particular use in paediatric studies where non-invasive tests are preferred, and where obtaining blood samples may prove difficult. The EIA also has several potential advantages over the UBT for population studies. Firstly, patients are not required to attend hospital as faecal specimens can be transported or posted to the laboratory. Secondly, no expensive instrumentation or expertise is required to perform a standard enzyme linked immunosorbent assay (ELISA) test. It is possible that delay between collection and analysis of stool might account for the lower sensitivity and specificity of the test that we have found compared to adult studies.

Our study did not include patients treated with antimicrobials or proton pump inhibitors, or those with diarrhoea. It is therefore not possible to comment on the effect that these factors may have on the EIA result. It is difficult to quantify the clinical significance of the cross reaction with $H$ acinonyx and $H$ felis. $H$ acinonyx is genetically very closely related to $H$ pylori, ${ }^{13}$ but there have been no reported cases of human infection. $H$ felis has been shown to cause human disease ${ }^{14}$ and there was speculation that domestic cats might play a role in the transmission of infection, ${ }^{15}$ but a recent study suggests that owning pets is not a risk factor. ${ }^{16}$

What, then, is the place of the EIA in the diagnosis and management of $H$ pylori infection? The definitive diagnosis of disease caused by $H$ pylori requires endoscopy. The UBT indicates the presence or absence of the organism in the stomach and is most appropriately used to confirm successful eradication of infection. With its high negative predictive value, ease of use, and non-invasive nature, the EIA is useful as a screening tool to exclude infection.

The detection of its antigens in stool supports the hypothesis that $H$ pylori is excreted in the faeces and might be the principle mode of transmission. However, stool antigen may be a product of digestion of the organism residing in the stomach. Therefore, other than two reports of culture from the stool, ${ }^{17}{ }^{18}$ there is little evidence that $H$ pylori survives passage through the gut. Nevertheless, detection of $H$ pylori antigens in the stool is likely to drive researchers with new determination to culture the organism from faeces, and to try to characterise the form in which it exists in, or passes through the large bowel. ${ }^{19}$ Therefore, in addition to its possible role as a diagnostic tool, the detection of stool antigens raises the possibility of genotyping the organism and thereby studying its transmission and epidemiology. ${ }^{19}$

We thank the Children's Research Fund for its support, the parents and children who made this study possible, and Dr Simon Ling for his very useful comments.

1 Banatvala N, Mayo K, Megraud F, Jennings R, Deeks JJ, Feldman RA. The cohort effect and Helicobacter pylori. Infect Dis 1993;168:219-21

2 Thomas J, Dale A, Harding M, Coward A, Cole TJ, Weaver LT. Helicobacter pylori colonisation in early life. Pediatr Res 1999;45:218-23.

3 Cullen DJE, Collins BJ, Christiansen KJ, Epis J, Warren JR, Surveyor I. When is Heliobacter pylori infection acquired? Gut 1993;34:1681-2.

4 Vaira D, Malfertheiner P, Megraud F, et al. HpSA European Study Group. Diagnosis of Helicobacter pylori infection with a new non-invasive antigen-based assay. Lancet 1999; 354:30-3.

5 Makristathis A, Pasching E, Schutze K, Wimmer M, Rotter ML, Hirschl AM. Detection of Helicobacter pylori in stool specimens by PCR and antigen EIA. 7 Clin Microbiol 1998; 36:2772-4.

6 Lehmann F, Drewe J, Terracciano L, Stuber R, Frei R, Beglinger C. Comparison of stool immunoassay with standard methods for detecting Helicobacter pylori infection. BMF 1999;319:1409

7 McNamara D, Whelan H, Hamilton H, Beattie S, O'Morain C. HpSA: assessment of a new non-invasive diagnostic assay for Helicobacter pylori infection in an Irish population. Ir F Med Sci 1999;168:111-13.

8 Chang MC, Wu MS, Wang HH, Wang HP, Lin JT. Helicobacter pylori stool antigen (HPSA) test-a simple, accurate and non-invasive test for detection of Helicobacter pylori infection. Hepatogastroenterology 1999;46:299-302.

9 Lopez-Brea M, Alarcon T, Megraud F. Diagnosis of Helicobacter pylori infection. Curr Opin Gastroenterol 1997;13:1319.

10 Thomas JE, Dale A, Harding $\mathrm{M}$, et al. Interpreting the ${ }^{13} \mathrm{C}-$ urea breath test among a large population of young hildren from a developing country. Pediatr Res 1999;46: 147-51

11 Eltumi M, Brueton MJ, Francis N. Diagnosis of Helicobacter pylori gastritis in children using the ${ }^{13} \mathrm{C}$ urea breath test. F Clin Gastroenterol 1999;28:238-40.

2 McNulty CAM, Nain P, Watson BE, Uff JS, Valori RM. A comparison of six serological kits for the diagnosis of Helicobacter pylori infection. Commun Dis Public Health 1999;2:59-64

13 Anderson LP, Boye K, Blom J, Holck S, Norgaard A, Elsborg L. Characterisation of a culturable "Gastropirilum hominis" (Helicobacter heilmannii) strain isolated from
human gastric mucosa. f Clin Microbiol 1999;37:1069-76.

14 Germani Y, Dauga C, Duval P, Huerre M, Levy M, Pialoux G, Sansonetti P, Grimont PA. Strategy for the detection of Helicobacter species by amplification of $16 \mathrm{~S}$ rRNA genes Helicobacter species by amplification of $16 \mathrm{~S}$ rRNA genes
and identificationof $\mathrm{H}$. felis in a human gastric biopsy. Res and identification of $\mathrm{H}$. felis in

15 Fox JG. Non-human reservoirs of Helicobacter pylori. Aliment Pharmacol Ther 1995;9:93-103.

16 Bode G. Pets are not a risk factor for Helicobacter pylori infection in young children: results of a population based study in Southern Germany. Pediatr Infect Dis $\mathcal{F}$ 1998;17: $909-12$

17 Thomas J, Gibson GR, Darboe MK, Dale A, Weaver LT. Isolation of Helicobacter pylori from human faeces. Lancet 1992;340:1194-5.

18 Kelly SM, Pitcher MCL, Farmery SM, Gibson GR. Isolation of Helicobacter pylori from feces of patients with dyspepsia in the United Kingdom. Gastroenterology 1994; 107:1671-4.

19 Weaver LT, Shepherd AJ, Williams C, Doherty CP, McColl KEL. Helicobacter pylori in the faeces? QfM 1999;92: $361-4$. 